\title{
A Robust Formation Control Strategy for Multi-Agent Systems with Uncertainties via Adaptive Gain Robust Controllers
}

\author{
Shun Ito ${ }^{1, *}$, Kaoru Ohara ${ }^{1}$, Yoshikatsu Hoshi ${ }^{1}$, Hidetoshi Oya $^{1}$, Shunya Nagai ${ }^{2}$ \\ ${ }^{1}$ Graduate School of Integrative Science and Engineering, Tokyo City University, Tokyo, Japan \\ ${ }^{2}$ Depertment of Information Systems Creation, Kanagawa University, Kanagawa, Japan \\ Received 05 December 2020; received in revised form 10 February 2021; accepted 28 February 2021
}

DOI: https://doi.org/10.46604/ijeti.2021.6825

\begin{abstract}
This paper deals with a design problem of an adaptive gain robust controller which achieves consensus for multi-agent system (MAS) with uncertainties. In the proposed controller design approach, the relative position between the leader and followers are considered explicitly, and the proposed adaptive gain robust controller consisting of fixed gains and variable ones tuned by time-varying adjustable parameters can reduce the effect of uncertainties. In this paper, we show that sufficient conditions for the existence of the proposed adaptive gain robust controller are reduced to solvability of linear matrix inequalities (LMIs). Finally, the effectiveness of the proposed robust formation control system is verified by simple numerical simulations. A main result of this study is that the proposed adaptive gain robust controller can achieve consensus and formation control giving consideration to relative distance in spite of uncertainties.
\end{abstract}

Keywords: multi-agent system (MAS), consensus, adaptive gain robust controller, relative distance, linear matrix inequalities (LMIs)

\section{Introduction}

For designing control systems for dynamical systems, it is necessary to derive a mathematical model for the controlled system. If the mathematical model represents the control system precisely, then the desired control system can be designed by various control design strategies. In particular, it is well known that LQ regulator for linear systems guarantees good robustness and asymptotic stability for closed-loop systems provided that its mathematical model can be described precisely [1-2]. However, it is unavoidable that there are some gaps between the original controlled system and its mathematical model, and these gaps are known as "uncertainty". Therefore, robust controller design methods have been well studied to deal with uncertainties explicitly, and a large number of robust control strategies have been proposed [3-8]. One can see that most of the conventional robust controllers have been designed by solving linear matrix inequalities (LMIs), and have consisted of the fixed gains designed by considering the worst-case variations for uncertainties. In addition to such typical robust control with fixed gains, adaptive gain robust controllers for uncertain systems have also been given [9-11]. Furthermore, comparing with the traditional fixed gain robust controller, these adaptive gain robust controllers are more flexible and adaptive.

Now, due to the rapid development of modern industries and sciences, controlled systems are highly complex and large in dimension, and such dynamical systems are referred to as "large-scale interconnected systems". Large-scale interconnected systems are characterized by a great multiplicity of measured outputs and combined inputs. Moreover, large-scale interconnected systems are defined as a group of interconnected subsystems such that decentralized operation is mandatory. For such large-scale interconnected systems, it is difficult to apply centralized control strategies. Thus, the design problems of

* Corresponding author. E-mail address: itustellar00@ gmail.com 
decentralized control for large-scale interconnected systems and the decentralized control strategies have been studied [12]. Furthermore, various design problems of decentralized robust control for uncertain large-scale interconnected systems have also been studied [13-16]. Additionally, various researchers have focused on formation control problems for multi-agent system (MAS) recently, and a multi-agent system can be defined as a network of a number of coupled dynamic units that are referred to agents. One can see that the design problems of formation control for MAS can be considered one of the decentralized control problems. Since consensus problem has the potential to be applied to various fields (for example, mobile robots, unmanned vehicles, vehicle formations, and so on), the consensus problem for design problems of formation control for MAS has been attracted a lot of attention. As is well known, consensus means that the states of all agents (for example, positions and velocities) are driven to a common state by applying distributed protocols. The consensus problem is often considered one of the most important and basic problem in formation control. Therefore, lots of existing results and researches for consensus problem for MAS have been well studied [17-24]. Olfati-Saber and Murray [17] considered the consensus problem for a network of first-order integrators with three different information network structures. Additionally, Xie et al. [18] analysed convergence for a consensus protocol for a class of networks of dynamic agents with fixed topology. Hence, there are many efficient results for the consensus problem. But in most cases, relative positions for each agent is not considered explicitly in the process of consensus. In addition, most of these results have dealt with a design problem of fixed gain controllers, and the derived controller has been designed by considering the worst-case variations of uncertainties/disturbances. Moreover, some results for consensus controllers based on event-triggered controller have also been reported [21-22]. However, there is no result for the adaptive gain controller designed by considering both uncertainties and relative positions of each agent explicitly. To bridge the gap, we have already presented a design method of an adaptive gain controller giving consideration to relative distances between agents [25]. The adaptive gain controller in our result [25] consists of fixed gains and variable ones tuned by time-varying adjustable parameters. Additionally, in the proposed control method, the upper bound on relative positions is the only information required, and the accurate information on the target position of other followers is nonessential.

From the above, on the basis of the existing result [25], this study handles a robust consensus problem for MAS with uncertainties. In the proposed robust control strategy, the proposed adaptive gain robust controller is composed of fixed gains and variable compensation inputs tuned by time-varying adjustable parameters. In this paper, we assume that the state equation for agents includes the information of the target relative position in advance, and sufficient conditions for the existence of the proposed adaptive gain robust controller can be reduced to solvability of LMIs. The advantages of the proposed adaptive gain robust controller are as follows: The proposed adaptive gain robust control strategy can achieve consensus robustly, and the proposed controller can be designed by solving LMIs, i.e. the proposed adaptive gain robust controller can easily be derived. Moreover, by introducing adaptive gains which can reduce the effects of uncertainties, consensus for MASs with uncertainties can robustly be achieved. Therefore, one can easily see that the proposed adaptive gain robust controller is a natural extension of our result [25]. Finally, simple numerical simulations are demonstrated to illustrate the effectiveness of the proposed formation control systems.

\section{Preliminaries}

This section shows the mathematical notation used in this paper. A list of mathematical symbols used in this paper is shown in Table 1 . For matrices $A \in \mathbb{R}^{m \times n}$ and $B \in \mathbb{R}^{p \times q}$, the Kronecker product $A \otimes B$ is defined as follows:

$$
A \otimes B \triangleq\left[\begin{array}{ccc}
a_{11} B & \cdots & a_{1 n} B \\
\vdots & \ddots & \vdots \\
a_{m 1} B & \cdots & a_{m n} B
\end{array}\right] .
$$


Moreover, we express the information path between agents based on graph theory $[17,26]$ in this paper. Graph theory is often used for control problems in MAS because the graph theory is useful for discussing the consensus problems. The graph is aggregation of vertices and edges, and the notation of a graph is $\mathcal{G} \in(\mathcal{V}, \varepsilon)$, where $\mathcal{V}=\{1,2, \cdots, N\}$ is the vertex set of $N$ vertices in the graph $\mathcal{G}$, and $\varepsilon$ is the edge set of connecting the vertices. In addition, $(i, j) \in \varepsilon$ means an edge between $i$ and $j$. Note that there are two types of graphs, undirected graph and directed one, and the graph is considered to be the directed graph in this paper. Furthermore, in order to express the graph algebraically, an adjacency matrix, a degree matrix $\mathcal{D}$, and a graph Laplacian $\mathcal{L}$ are introduced in this paper. The adjacency matrix $\mathcal{A}$ represents the adjacency relation of each vertex of the graph. In the graph $\mathcal{G} \in(\mathcal{V}, \varepsilon)$, when there is an directed edge from $j \in \mathcal{V}$ to $i \in \mathcal{V}$ for a pair $(i, j) \in \varepsilon$, i.e. there exists a available edges from $j$ to $i$, the vertex $i$ is said to be adjacent to vertex $j$. In this case, the adjacent set of vertices $i$ is $\mathcal{N}_{i} \triangleq$ $\{j \in \mathcal{V} \mid(i, j) \in \varepsilon\}$, and the elements $a_{i j}$ for the adjacency matrix $\mathcal{A}=\left[a_{i j}\right]$ is defined as:

$$
a_{i j} \triangleq \begin{cases}1 & \text { if }(i, j) \in \mathcal{E} \text { and } i \neq j \\ 0 & \text { else }\end{cases}
$$

Additionally, in the directed graph $\mathcal{G} \in(\mathcal{V}, \varepsilon)$, the in-degree of a vertex $i$ represents the number of edges incoming to the vertex $i$ and it is denoted as $d_{i}^{i n}$. By the way, out-degree means the number of edges outgoing from a vertex. Then for the graph $\mathcal{G} \in(\mathcal{V}, \varepsilon)$, the degree matrix $\mathcal{D} \in \mathbb{R}^{N \times N}$ are defined as:

$$
\mathcal{D}=\operatorname{diag}\left(d_{1}^{\text {in }}, d_{2}^{\text {in }}, \cdots, d_{N}^{\text {in }}\right)
$$

Additionally, the graph Laplacian $\mathcal{L}=\left[l_{i j}\right]$ are defined as:

$$
\mathcal{L}=\mathcal{D}-\mathcal{A}
$$

One can see that the graph Laplacian $\mathcal{L}$ represents the structure of the graph (i.e., the in-degree of each vertex and the relationship for edges) in a single matrix. Therefore, in the consensus problem for MAS, the graph Laplacian $\mathcal{L}$ is utilized for describing features of MASs.

Table 1 Mathematical symbols in this paper

\begin{tabular}{|c|c|}
\hline $\mathbb{R}^{m \times n}$ & $m$ by $n$ real matrix \\
\hline$I_{n}$ & $n$-dimensional identity matrix \\
\hline$A>0(A \geq 0)$ & The matrix $A$ is positive definite (positive semi definite) \\
\hline$A<0(A \leq 0)$ & The matrix $A$ is negative definite (negative semi definite) \\
\hline$\|a\|$ & Euclidean norm of a vector $a$ \\
\hline$\|C\|$ & The matrix norm of any matrix $C$ induced by the vector norm \\
\hline $\operatorname{diag}\left(A_{1}, A_{2}, \cdots, A_{n}\right)$ & Diagonal block matrix with matrices $A_{i}(i=1,2, \cdots, n)$ \\
\hline$*$ & Symmetric element of matrix \\
\hline$A \otimes B$ & Kronecker product \\
\hline
\end{tabular}

\section{Problem Formulation}

In this paper, we consider the multi-agent systems (MAS) consisting of $N$ agents with leader-follower structure. "Leader-follower structure" is a kind of MASs composed by defining an agent as a leader and the other agents (followers). Furthermore, it is assumed that the agent's dynamics includes the structured uncertainties. Namely, each agent is represented by the following state equation:

$$
\frac{d}{d t} \bar{x}_{i}(t)=(A+B \Delta(t) E) \bar{x}_{i}(t)+B u_{i}(t)
$$


where $A \in \mathbb{R}^{n \times n}, B \in \mathbb{R}^{n \times m}$ and $E \in \mathbb{R}^{p \times n}$ are known constant matrices, and $\Delta(t) \in \mathbb{R}^{m \times p}$ is an unknown time-varying matrix satisfying $\|\Delta(t)\| \leq 1.0$. It is well known that the uncertain term $B \Delta(t) E$ is referred to as "structured uncertainties", and the uncertainties in the system satisfy the matching condition [4]. Moreover, a new state variable $\bar{x}_{i}(t)\left(\bar{x}_{i}(t) \triangleq x_{i}(t)-d_{i}\right)$ for the difference between the state of each agent $x_{i}(t)$ and the target relative position $d_{i}$ from the leader to the $i$-th follower is defined. It is assumed that the information about the relative position between the leader and other followers cannot be obtained by the $i$-th follower, but can be obtained by the upper bound of the relative position $d_{m i}$ which satisfies $\left\|d_{i}\right\| \leq d_{m i}$.

Now, we consider the control input $u_{i}(t)$ for each agent. Firstly, the following state feedback input is defined as:

$$
u_{K i}(t)=K \bar{x}_{i}(t)
$$

where $K \in \mathbb{R}^{m \times n}$ is a feedback gain matrix which stabilizes the matrix $A_{K} \triangleq A+B K$ [24]. Moreover, in order to achieve consensus [25-26], we introduce the following input $u_{F i}(t)$ as:

$$
u_{F i}(t)=F \sum_{j \in \mathcal{N}_{i}}\left(x_{i}(t)-x_{j}(t)\right)
$$

In Eq. (7), $F \in \mathbb{R}^{m \times n}$ is a consensus gain, and for $i$-th agent, we define the following control input $u_{i}(t)$ :

$$
\begin{aligned}
u_{i}(t) & \triangleq u_{K i}(t)+u_{F i}(t)+v_{i}(t) \\
& =K \bar{x}_{i}(t)+F \sum_{j \in \mathcal{N}_{i}}\left(x_{i}(t)-x_{j}(t)\right)+v_{i}(t)
\end{aligned}
$$

where $v_{i}(t) \in \mathbb{R}^{m}$ is a compensation input [25] for reducing the effects of relative position and uncertainties. Then, by introducing the augmented vectors $\bar{x}(t)=\left(\bar{x}_{1}^{T}(t), \bar{x}_{2}^{T}(t), \cdots, \bar{x}_{N}^{T}(t)\right)^{T}$ and $u(t)=\left(u_{1}^{T}(t), u_{2}^{T}(t), \cdots, u_{N}^{T}(t)\right)^{T}$ we obtain:

$$
\frac{d}{d t} \bar{x}(t)=\left(I_{N} \otimes \bar{A}\right) \bar{x}(t)+\left(I_{N} \otimes B\right) u(t)
$$

In Eq. (9), $\bar{A}$ is a matrix given by $\bar{A}=A+B \Delta(t) E$. Furthermore, from Eqs. (8) and (9), one can derive:

$$
\begin{aligned}
\frac{d}{d t} \bar{x}(t) & =\left(I_{N} \otimes \bar{A}\right) \bar{x}(t)+\left(I_{N} \otimes B\right) u(t) \\
& =\left[\bar{A}_{N}+B_{N}\left\{K_{N}+(\mathcal{L} \otimes F)\right\}\right] \bar{x}(t)+B_{N}\{(\mathcal{L} \otimes F) d+v(t)\}
\end{aligned}
$$

where matrices $\bar{A}_{N}, B_{N}$, and $K_{N}$ are given by $\bar{A}_{N} \triangleq I_{N} \otimes \bar{A}, B_{N} \triangleq I_{N} \otimes B$, and $K_{N} \triangleq I_{N} \otimes K$, respectively. Moreover, the vectors $d$ and $v(t)$ in Eq. (10) are defined as $d \triangleq\left(d_{1}^{T}, d_{2}^{T}, \cdots, d_{N}^{T}\right)^{T}, v(t)=\left(v_{1}^{T}(t), v_{2}^{T}(t), \cdots, v_{N}^{T}(t)\right)^{T}$, respectively.

From the above discussion, the controller design objective in this paper is to design the stabilizing feedback gain $K$, the consensus gain $F$, and compensation input $v(t)$ such that asymptotical stability of the closed-loop system of Eq. (10) is guaranteed.

\section{Main Results}

This chapter shows an LMI-based design method of the stabilizing feedback gain $K$, the consensus gain $F$, and compensation input $v(t)$ for the multi-agent system. In the following, we assume the number of agents is 3 , i.e. $N=3$, for simplicity. Moreover, in this study, the first agent is the leader $i=1=$ " $l$ ", and the other agents mean followers. And, the graph of network topology between agents is assumed as Fig. 1. Then, the adjacency matrix $\mathcal{A}$, the degree matrix $\mathcal{D}$, and the graph Laplacian $\mathcal{L}$ can be obtained as follows. 


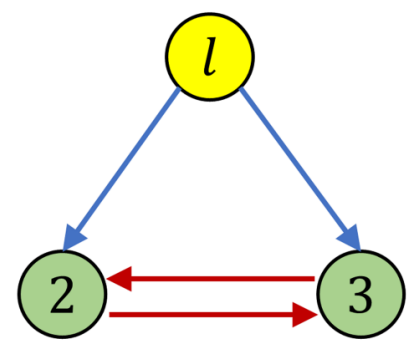

Fig.1 The graph corresponding to MAS consisting of three agents

$$
\mathcal{A}=\left(\begin{array}{lll}
0 & 0 & 0 \\
1 & 0 & 1 \\
1 & 1 & 0
\end{array}\right), \quad \mathcal{D}=\left(\begin{array}{lll}
0 & 0 & 0 \\
0 & 2 & 0 \\
0 & 0 & 2
\end{array}\right), \quad \mathcal{L}=\left(\begin{array}{ccc}
0 & 0 & 0 \\
-1 & 2 & -1 \\
-1 & -1 & 2
\end{array}\right)
$$

Note that the proposed design method can easily be extended the case of multi-agent systems consisting of 4 or more agents.

Now, we give the following theorem for determining the stabilizing feedback gain $K$, the consensus gain $F$, and compensation input $v(t)$ for the closed-loop system of Eq. (10).

Theorem 1: Consider the closed-loop system of Eq. (10) in which the control input of Eq. (8) is applied to the multi-agent system of Eq. (9). If there exist solutions $S \in \mathbb{R}^{n \times n}, W_{K} \in \mathbb{R}^{m \times n}$, and $W_{F} \in \mathbb{R}^{m \times n}$ of the following linear matrix inequality (LMI) condition:

$$
\begin{aligned}
& \left(\begin{array}{ccc}
\Psi_{11}\left(S, W_{K}\right) & \Psi_{12}\left(W_{F}\right) & \Psi_{13}\left(W_{F}\right) \\
\star & \Psi_{22}\left(S, W_{K}, W_{F}\right) & \Psi_{23}\left(W_{F}\right) \\
\star & \star & \Psi_{33}\left(S, W_{K}, W_{F}\right)
\end{array}\right)<0 \\
& \Psi_{11}\left(S, W_{K}\right)=S A^{T}+W_{K}^{T} B^{T}+A S+B W_{K} \\
& \Psi_{22}\left(S, W_{K}, W_{F}\right)=\Psi_{33}\left(S, W_{K}, W_{F}\right)=S A^{T}+W_{K}^{T} B^{T}+2 W_{F}^{T} B^{T}+A S+B W_{K}+2 B W_{F} \\
& \Psi_{12}\left(W_{F}\right)=\Psi_{13}\left(W_{F}\right)=-W_{F}^{T} B^{T}, \Psi_{23}\left(W_{F}\right)=-\left(W_{F}^{T} B^{T}+B W_{F}\right)
\end{aligned}
$$

then asymptotical stability of the closed-loop system of Eq. (10) is ensured, and the consensus for MAS of Eq. (9) can be achieved. In addition, the stabilizing feedback gain $K \in \mathbb{R}^{m \times n}$ and the consensus gain $F \in \mathbb{R}^{m \times n}$ can be calculated by using solutions $S \in \mathbb{R}^{n \times n}, W_{K} \in \mathbb{R}^{m \times n}$, and $W_{F} \in \mathbb{R}^{m \times n}$ as follows:

$$
K=W_{K} S^{-1}, F=W_{F} S^{-1}
$$

and by introducing the matrix $P=S^{-1}$, the compensation inputs $v_{i}(t)$ are designed as follows:

$$
\left\{\begin{array}{l}
v_{l}(t)=-\frac{\left\|B^{T} P \bar{x}_{l}(t)\right\|\left\|E \bar{x}_{l}(t)\right\|}{\left\|B^{T} P \bar{x}_{l}(t)\right\|^{2}} B^{T} P \bar{x}_{l}(t) \\
v_{i}(t)=-2 F d_{i}-\frac{d_{m j}\left\|F^{T} B^{T} P \bar{x}_{i}(t)\right\|+\left\|B^{T} P \bar{x}_{i}(t)\right\|\left\|E \bar{x}_{i}(t)\right\|}{\left\|B^{T} P \bar{x}_{i}(t)\right\|^{2}} B^{T} P \bar{x}_{i}(t) \quad(i=2,3)
\end{array}\right.
$$

Proof 1: Now, we introduce the following Lyapunov function candidate;

$$
\mathcal{V}(\bar{x}, t)=\bar{x}^{T}(t)\left(I_{3} \otimes P\right) \bar{x}(t)
$$

where $P \in \mathbb{R}^{n \times n}$ is a symmetric positive definite matrix. The time derivative of Eq. (15) along the trajectory of the closed-loop system of Eq. (10) can be written as: 


$$
\begin{aligned}
\frac{d}{d t} V(\bar{x}, t) & =\left(\left[A_{3}+B_{3}\left\{K_{3}+(\mathcal{L} \otimes F)\right\}\right] \bar{x}(t)+B_{3}\left\{(\mathcal{L} \otimes F) d(t)+\left(I_{3} \otimes \Delta(t) E\right) \bar{x}(t)+v(t)\right\}\right)^{T} P_{3} \bar{x}(t) \\
& +\bar{x}^{T}(t) P_{3}\left(\left[A_{3}+B_{3}\left\{K_{3}+(\mathcal{L} \otimes F)\right\}\right] \bar{x}(t)+B_{3}\left\{(\mathcal{L} \otimes F) d+\left(I_{3} \otimes \Delta(t) E\right) \bar{x}(t)+v(t)\right\}\right) \\
& =\bar{x}^{T}(t)\left[A_{3}+B_{3}\left\{K_{3}+(\mathcal{L} \otimes F)\right\}\right]^{T} P_{3} \bar{x}(t)+\bar{x}^{T}(t) P_{3}\left[A_{3}+B_{3}\left\{K_{3}+(\mathcal{L} \otimes F)\right\}\right] \bar{x}(t) \\
& +\bar{x}^{T}(t)\left[P_{3} B_{3}\left\{(\mathcal{L} \otimes F) d+\left(I_{3} \otimes \Delta(t) E\right) \bar{x}(t)+v(t)\right\}\right] \\
& +\left[P_{3} B_{3}\left\{(\mathcal{L} \otimes F) d+\left(I_{3} \otimes \Delta(t) E\right) \bar{x}(t)+v(t)\right\}\right]^{T} \bar{x}(t) \\
& =\bar{x}(t)^{T} \Phi(P, K, F) \bar{x}(t)+2 \omega(P, F, v(t))
\end{aligned}
$$

In Eq. (16), $\Phi(P, K, F)$ and $\omega(P, F, v(t))$ are the matrix and the scalar represented by:

$$
\begin{aligned}
\Phi(P, K, F)= & {\left[A_{3}+B_{3}\left\{K_{3}+(\mathcal{L} \otimes F)\right\}\right]^{T} P_{3}+P_{3}\left[A_{3}+B_{3}\left\{K_{3}+(\mathcal{L} \otimes F)\right\}\right] } \\
\omega(P, F, v(t))= & {\left[P_{3} B_{3}\left\{(\mathcal{L} \otimes F) d+\left(I_{3} \otimes \Delta(t) E\right) \bar{x}(t)+v(t)\right\}\right]^{T} \bar{x}(t) } \\
& =\left(\begin{array}{c}
\bar{x}_{l}^{T}(t) E^{T} \Delta^{T}(t) B^{T} P \bar{x}_{l}(t)+v_{l}^{T}(t) B^{T} P \bar{x}_{l}(t) \\
\left(2 d_{2}^{T}-d_{3}^{T}\right) F^{T} B^{T} P \bar{x}_{2}(t)+\bar{x}_{2}^{T}(t) E^{T} \Delta^{T}(t) B^{T} P \bar{x}_{2}(t)+v_{2}^{T}(t) B^{T} P \bar{x}_{2}(t) \\
\left(2 d_{3}^{T}-d_{2}^{T}\right) F^{T} B^{T} P \bar{x}_{3}(t)+\bar{x}_{3}^{T}(t) E^{T} \Delta^{T}(t) B^{T} P \bar{x}_{3}(t)+v_{3}^{T}(t) B^{T} P \bar{x}_{3}(t)
\end{array}\right)
\end{aligned}
$$

where $P_{3}=\left(I_{3} \otimes P\right)$. From $\|\Delta(t)\| \leq 1.0$ and $\left\|d_{i}\right\| \leq d_{m i}$, one can easily see that the relation holds:

$$
\begin{aligned}
d_{j}^{T} F^{T} B^{T} P \bar{x}_{i}(t)+\bar{x}_{i}^{T}(t) E^{T} \Delta^{T}(t) B^{T} P \bar{x}_{i}(t) & \leq\left\|d_{j}^{T} F^{T} B^{T} P \bar{x}_{i}(t)\right\|+\left\|\bar{x}_{i}^{T}(t) E^{T} \Delta^{T}(t) B^{T} P \bar{x}_{i}(t)\right\| \\
& \leq\left\|d_{j}\right\|\left\|F^{T} B^{T} P \bar{x}_{i}(t)\right\|+\left\|\Delta^{T}(t)\right\|\left\|B^{T} P \bar{x}_{i}(t)\right\|\left\|E \bar{x}_{i}(t)\right\| \\
& \leq d_{m j}\left\|F^{T} B^{T} P \bar{x}_{i}(t)\right\|+\left\|B^{T} P \bar{x}_{i}(t)\right\|\left\|E \bar{x}_{i}(t)\right\|
\end{aligned}
$$

Thus, we select the following compensation input $v_{i}(t)$ :

$$
\left\{\begin{array}{l}
v_{l}(t)=-\frac{\left\|B^{T} P \bar{x}_{l}(t)\right\|\left\|E \bar{x}_{l}(t)\right\|}{\left\|B^{T} P \bar{x}_{l}(t)\right\|^{2}} B^{T} P \bar{x}_{l}(t) \\
v_{i}(t)=-2 F d_{i}-\frac{d_{m j}\left\|F^{T} B^{T} P \bar{x}_{i}(t)\right\|+\left\|B^{T} P \bar{x}_{i}(t)\right\|\left\|E \bar{x}_{i}(t)\right\|}{\left\|B^{T} P \bar{x}_{i}(t)\right\|^{2}} B^{T} P \bar{x}_{i}(t) \quad(i=2,3)
\end{array}\right.
$$

If the compensation input $v_{i}(t)$ of Eq. (20) is selected, then the following relation holds:

$$
\frac{d}{d t} \mathcal{V}(\bar{x}, t) \leq \bar{x}(t)^{T} \Phi(P, K, F) \bar{x}(t)
$$

where the matrix $\Phi(P, K, F)$ is expressed as:

$$
\Phi(P, K, F)=\left(\begin{array}{ccc}
\Phi_{11}(P, K) & \Phi_{12}(P, F) & \Phi_{13}(P, F) \\
\star & \Phi_{22}(P, K, F) & \Phi_{23}(P, F) \\
\star & \star & \Phi_{33}(P, K, F)
\end{array}\right)
$$

where $\Phi_{i j}(P, K, F)$ can be written as follows: 


$$
\begin{aligned}
& \Phi_{11}(P, K)=\left(A^{T}+K^{T} B^{T}\right) P+P(A+B K) \\
& \Phi_{22}(P, K, F)=\Phi_{33}(P, K, F)=\left(A^{T}+K^{T} B^{T}+2 F^{T} B^{T}\right) P+P(A+B K+2 B F) \\
& \Phi_{12}(P, F)=\Phi_{13}(P, F)=-F^{T} B^{T} P \\
& \Phi_{23}(P, F)=-F^{T} B^{T} P-P B F
\end{aligned}
$$

Therefore, if the inequality condition is satisfied as:

$$
\Phi(P, K, F)<0
$$

then the following inequality for the function $\mathcal{V}(\bar{x}, t)$ is obtained:

$$
\Phi(P, K, F)<0 \frac{d}{d t} \mathcal{V}(\bar{x}, t)<0
$$

That is, the function $\mathcal{V}(\bar{x}, t)$ of Eq. (15) becomes a Lyapunov function of the closed-loop system of Eq. (10), and the asymptotic stability of the closed-system of Eq. (10) is guaranteed.

Now, we consider the inequality condition of Eq. (24). Here, in order to determine the feedback gain $K \in \mathbb{R}^{m \times n}$ and the consensus gain $F \in \mathbb{R}^{m \times n}$, a symmetric positive definite matrix $S=P^{-1}$ and complementary variables $W_{K} \triangleq K S$ and $W_{F} \triangleq F S$ are introduced. Moreover, pre- and post-multiplying Eq. (24) by $S_{3}=I_{3} \otimes S$, we obtain:

$$
S_{3} \Phi(P, K, F) S_{3}=\left(\begin{array}{ccc}
\Psi_{11}\left(S, W_{K}\right) & \Psi_{12}\left(W_{F}\right) & \Psi_{13}\left(W_{F}\right) \\
\star & \Psi_{22}\left(S, W_{K}, W_{F}\right) & \Psi_{23}\left(W_{F}\right) \\
\star & \star & \Psi_{33}\left(S, W_{K}, W_{F}\right)
\end{array}\right)<0
$$

In Eq. (26), $\psi_{i j}\left(S, W_{K}, W_{F}\right)$ is expressed as;

$$
\begin{aligned}
& \Psi_{11}\left(S, W_{K}\right)=S A^{T}+W_{K}^{T} B^{T}+A S+B W_{K} \\
& \Psi_{22}\left(S, W_{K}, W_{F}\right)=\Psi_{33}\left(S, W_{K}, W_{F}\right)=S A^{T}+W_{K}^{T} B^{T}+2 W_{F}^{T} B^{T}+A S+B W_{K}+2 B W_{F} \\
& \Psi_{12}\left(W_{F}\right)=\Psi_{13}\left(W_{F}\right)=-W_{F}^{T} B^{T} \\
& \Psi_{23}\left(W_{F}\right)=-\left(W_{F}^{T} B^{T}+B W_{F}\right)
\end{aligned}
$$

Thus, one can see that the inequality of Eq. (26) is a linear matrix inequality (LMI) for $S \in \mathbb{R}^{n \times n}, W_{K} \in \mathbb{R}^{m \times n}$, and $W_{F} \in$ $\mathbb{R}^{m \times n}$. If the solution of Eq. (26) exists, then asymptotical stability of the closed-loop system of Eq. (10) is guaranteed. Moreover, by using $S \in \mathbb{R}^{n \times n}, W_{K} \in \mathbb{R}^{m \times n}$, and $W_{F} \in \mathbb{R}^{m \times n}$, which satisfy the LMI of Eq. (26), the gains $K$ and $F$ can be derived as:

$$
K=W_{K} S^{-1}, F=W_{F} S^{-1}
$$

From the above discussion, the proof of Theorem 1 is completed.

Remark 1: If the solutions $S \in \mathbb{R}^{n \times n}, W_{K} \in \mathbb{R}^{m \times n}$, and $W_{F} \in \mathbb{R}^{m \times n}$ of the LMI of Eq. (12) cannot be obtained, the internal stability of closed-loop system is not ensured and the consensus cannot be achieved, i.e. the proposed adaptive gain robust controller cannot be constructed. In other words, accomplishment of our control objective in the proposed formation control strategy is reduced to the solvability of LMIs. Additionally, one can see that the LMI of Eq. (12) is equivalent to one in Theorem 1 of our previous work [25]. Thus, the stabilizing feedback gain $K$ and the consensus gain $F$ in the proposed robust formation control are same as ones in [25] and only the compensation input is different. From this fact, one can see that the result of this paper is a natural extension of the existing result [25]. 


\section{Numerical Simulations}

In this section, simple numerical simulations are given to show the effectiveness of the formation control system with the proposed adaptive gain robust controller.

In this paper, the simulation results for three cases (Case 1-3) are provided to illustrate the effectiveness of the proposed formation control systems. In Case 1, the simulation results of the formation control via the proposed adaptive gain robust controller is shown. Moreover, we compare the proposed formation control strategy with the existing result [25] in which the relative distances are considered only, and the existing result [25] is shown in Case 2. Additionally, Case 3 represents the results for the "fixed" gain robust controller (see Appendix). Note that the numerical simulations for all cases have been done under the same conditions. Furthermore, in the case of the "fixed" gain robust controller, the exact information for relative positions is required (see Remark A.1 in Appendix), and thus in this example, it is supposed that the exact information for relative positions can be obtained.

Firstly, we consider agents represented by the following state equation:

$$
\frac{d}{d t} x_{i}(t)=\left(\begin{array}{cccc}
0 & 1 & 0 & 0 \\
-1.75 \delta_{1}(t) & 1.75 \delta_{1}(t) & 0 & 1.75 \delta_{1}(t) \\
0 & 0 & 0 & 1 \\
0 & 0 & 0 & 1.75 \delta_{2}(t)
\end{array}\right) x_{i}(t)+\left(\begin{array}{ll}
0 & 0 \\
1 & 0 \\
0 & 0 \\
0 & 1
\end{array}\right) u_{i}(t)
$$

where $\delta_{1}(t)$ and $\delta_{2}(t)$ are unknown parameters and are set as $\Delta(t)=\operatorname{diag}\left(\delta_{1}(t), \delta_{2}(t)\right)$. Furthermore, $\delta_{1}(t)$ and $\delta_{2}(t)$ are given by:

$$
\begin{aligned}
& \delta_{1}(t)=1.0 \exp (-0.05 t) \sin (10 \pi t) \\
& \delta_{2}(t)=1.0 \exp (-0.05 t) \cos (10 \pi t)
\end{aligned}
$$

From Eq. (29), the matrix $E$ can be described as:

$$
E=\left(\begin{array}{cccc}
-1.75 & 1.75 & 0 & 1.75 \\
0 & 0 & 0 & 1.75
\end{array}\right)
$$

Moreover, it is supposed that the state variable $x_{i}(t)$ is given by $x_{i}(t)=\left(x_{i}^{(1)}(t) x_{i}^{(2)}(t) x_{i}^{(3)}(t) x_{i}^{(4)}(t)\right)^{T}$. For the state variable in Eq. (29), $x_{i}^{(1)}(t)$ and $x_{i}^{(2)}(t)$ (resp. $x_{i}^{(3)}(t)$ and $\left.x_{i}^{(4)}(t)\right)$ are the position and velocity in the $x$-axis (resp. $y$-axis). see the existing result [25].

By solving LMI of Eq. (26), the following matrices are obtained:

$$
\begin{aligned}
& S=\left(\begin{array}{cccc}
1.1574 & -4.2577 \times 10^{-1} & 2.0310 \times 10^{-3} & -3.4560 \times 10^{-3} \\
\star & 1.1574 & 3.4550 \times 10^{-3} & 2.0330 \times 10^{-3} \\
\star & \star & 1.1574 & -4.2577 \times 10^{-1} \\
\star & \star & \star & 1.1574
\end{array}\right) \\
& W_{K}=\left(\begin{array}{ccc}
-1.1651 & -9.708 \times 10^{-3} \\
-1.9053 & -2.1926 \\
-9.7081 \times 10^{-3} & -1.1651 \\
-1.6958 \times 10^{-18} & -1.9053
\end{array}\right)^{T}
\end{aligned}
$$


Therefore, the stabilizing feedback gain $K \in \mathbb{R}^{2 \times 4}$ and the consensus gain $F \in \mathbb{R}^{2 \times 4}$ for Case 1 and Case 2 can be calculated as:

$$
\begin{aligned}
K & =\left(\begin{array}{crcc}
-1.8646 & -2.3322 & -1.4535 \times 10^{-2} & -6.8409 \times 10^{-3} \\
-8.2057 \times 10^{-1} & -2.1978 & -1.8699 & -2.3327
\end{array}\right) \\
F & =\left(\begin{array}{llll}
2.9273 & 7.9213 & 2.1088 & 5.6819 \\
2.1088 & 5.6819 & 2.9274 & 7.9213
\end{array}\right) \times 10^{-1}
\end{aligned}
$$

Next, in order to determine the gains $K \in \mathbb{R}^{2 \times 4}$ and $F \in \mathbb{R}^{2 \times 4}$ for the "fixed" gain robust controller (Case 3), we solve the LMI of Eq. (A.14) in Theorem A.1 in Appendix. Then, the matrices $S, W_{K}$, and $W_{F}$ and the positive scalar $\gamma$ are obtained as:

$$
\begin{aligned}
& S=\left(\begin{array}{cccc}
32.0270 & -34.3991 & -46.2393 & 25.2218 \\
\star & 70.1974 & -4.3522 & -31.8467 \\
\star & \star & 331.5794 & -51.0899 \\
\star & \star & \star & 61.8142
\end{array}\right) \\
& W_{K}=\left(\begin{array}{ccc}
-33.9315 & 18.7995 \\
-295.7166 & 15.8179 \\
23.6860 & -32.6215 \\
1.3072 \times 10^{-18} & -282.8710
\end{array}\right)^{T}, W_{F}=\left(\begin{array}{cc}
-3.5769 \times 10^{-7} & -2.0300 \times 10^{-5} \\
-3.4951 \times 10^{-4} & -2.5277 \times 10^{-5} \\
-4.2221 \times 10^{-5} & 5.7594 \times 10^{-5} \\
3.0623 \times 10^{-5} & -3.5272 \times 10^{-4}
\end{array}\right)^{T} \\
& \gamma=322.1627
\end{aligned}
$$

Therefore, the gain matrices $K \in \mathbb{R}^{2 \times 4}$ and $F \in \mathbb{R}^{2 \times 4}$ for the "fixed" gain robust controller can be derived as:

$$
\begin{aligned}
K & =\left(\begin{array}{cccc}
-21.4083 & -16.0091 & -3.4940 & -2.4006 \\
4.4184 & -1.0228 & -0.6414 & -7.4360
\end{array}\right) \\
F & =\left(\begin{array}{llll}
-2.1740 \times 10^{-5} & -1.7005 \times 10^{-5} & -3.7690 \times 10^{-6} & -2.5100 \times 10^{-6} \\
-9.2678 \times 10^{-7} & -5.0285 \times 10^{-6} & -1.4229 \times 10^{-6} & -9.0947 \times 10^{-6}
\end{array}\right)
\end{aligned}
$$

In this example, the initial values of each agent are selected as follows:

$$
x_{l}(0)=\left(\begin{array}{llll}
5.0 & -2.0 & 3.0 & 2.0
\end{array}\right)^{T}, x_{2}(0)=\left(\begin{array}{llll}
6.0 & 4.0 & 0.0 & 3.0
\end{array}\right)^{T}, x_{3}(0)=\left(\begin{array}{llll}
1.0 & 2.0 & 2.0 & 4.0
\end{array}\right)^{T}
$$

Furthermore, the desired relative position of the leader and follower $d_{2}$ and $d_{3}$ are set as:

$$
d_{2}=\left(\begin{array}{llll}
-2.0 & 0.0 & -2.0 & 0.0
\end{array}\right)^{T}, d_{3}=\left(\begin{array}{llll}
2.0 & 0.0 & -2.0 & 0.0
\end{array}\right)^{T}
$$

Additionally, $d_{m 2}$ and $d_{m 3}$ are set as $d_{m 2}=d_{m 3}=2 \sqrt{2}$. Also, we introduce $d_{l}(t)$ as the reference signal to the reader $r(t)$ expressed by the following.

$$
r(t)=\left(\begin{array}{c}
3.0 \cos \left(1.0 \times 10^{-1} \pi t\right) \\
-3.0 \times 10^{-1} \pi \sin \left(1.0 \times 10^{-1} \pi t\right) \\
3.0 \sin \left(1.0 \times 10^{-1} \pi t\right) \\
3.0 \times 10^{-1} \pi \cos \left(1.0 \times 10^{-1} \pi t\right)
\end{array}\right)
$$

The reference signal of Eq. (36) means that the reader draws a circular orbit with a radius of 3.0 around the origin, and the followers will behave the desired trajectory according to the specified relative position. 
The results of this numerical simulation for Case 1 are shown in Figs. 2-6. In addition, Figs. 2-5 represents the time histories of $x_{i}^{(1)}(t) \sim x_{i}^{(4)}(t)$ for each agent, respectively. From the results of Figs. 2-5, the leader agent follows the given reference signal in spite of uncertainties, and followers with unknown parameters track the desired trajectory corresponding to the specified relative positions, respectively. Fig. 6 shows the state trajectories of each agent and depicts the shape of formations, i.e. Fig. 6 represents the movement of each agent on the $x-y$ coordinates. From these figures, we can see that each agent forms a desired formation. Therefore, it can be confirmed that the consensus has robustly been achieved by the proposed adaptive gain robust controller, and the effectiveness of the proposed formation control strategy is shown.

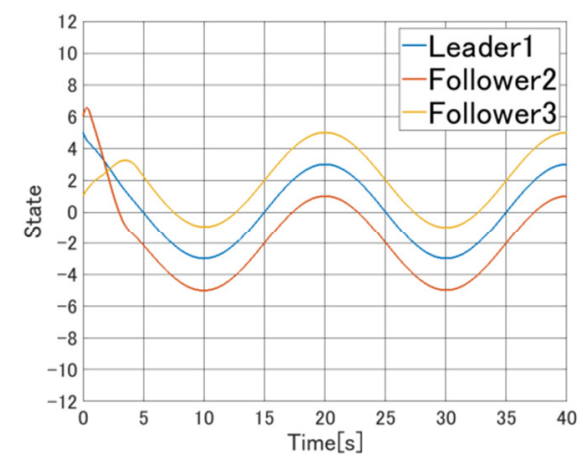

Fig. 2 Time-histories of $x_{i}^{(1)}(t)$ in Case 1

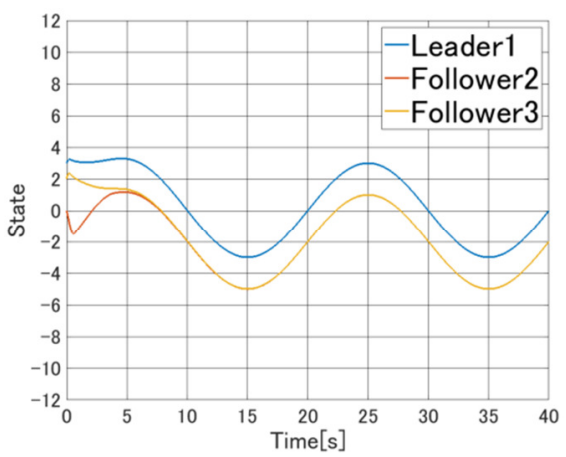

Fig. 4 Time-histories of $x_{i}^{(3)}(t)$ in Case 1

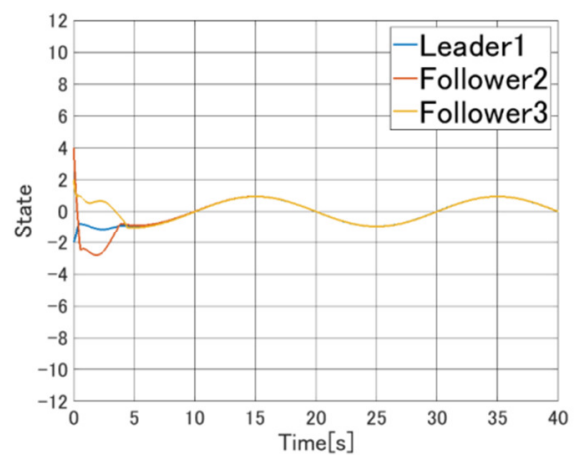

Fig. 3 Time-histories of $x_{i}^{(2)}(t)$ in Case 1

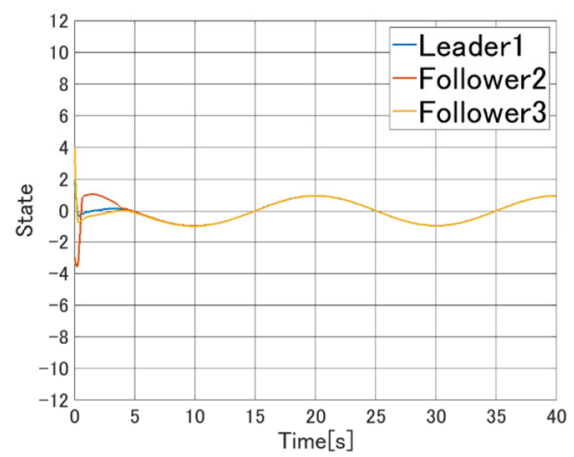

Fig. 5 Time-histories of $x_{i}^{(4)}(t)$ in Case 1
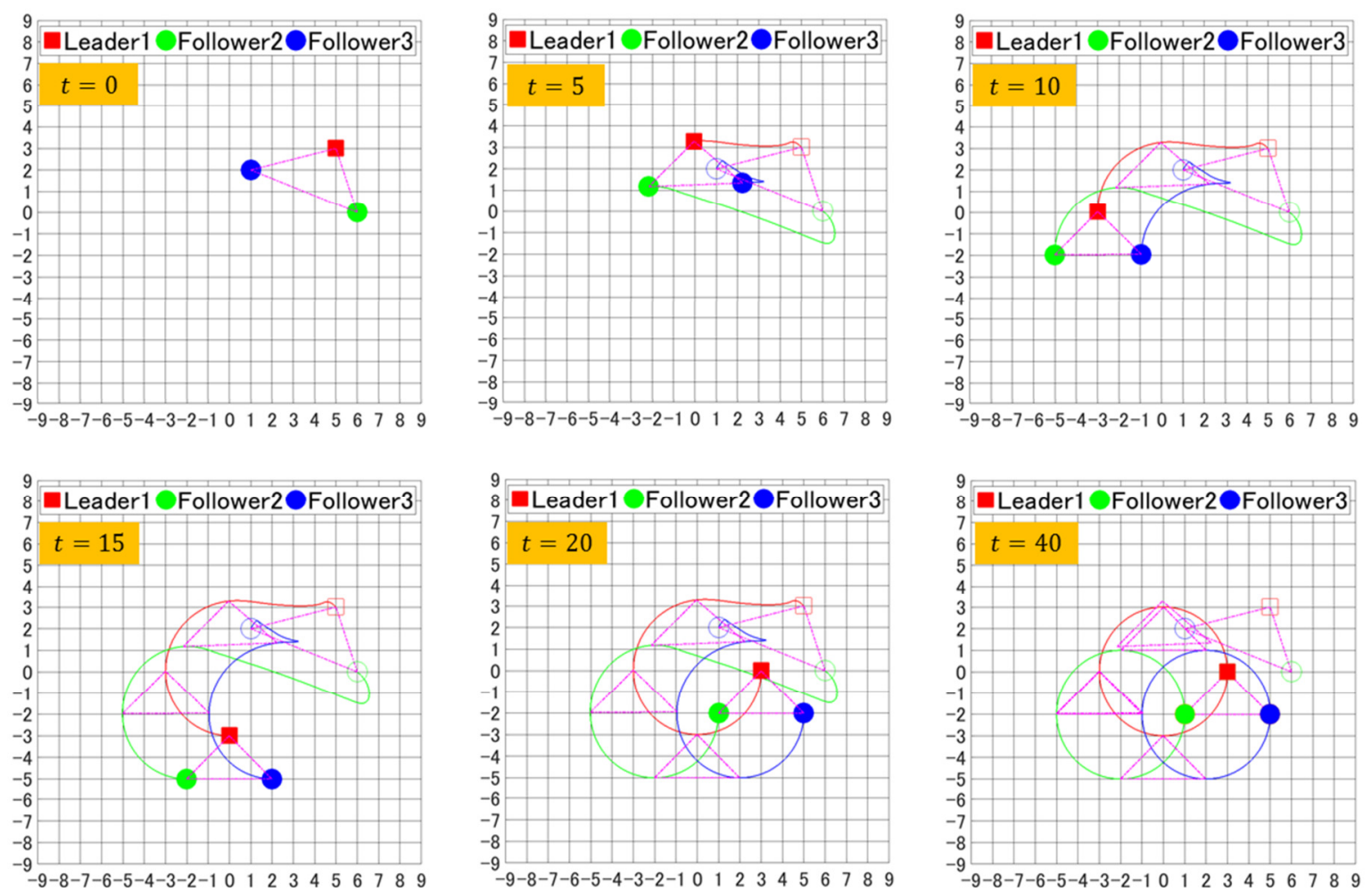

Fig. 6 Trajectories $(x-y$ plane) of MASs in Case 1 
The results for Case 2 are shown as Figs. 7-11, representing that the time histories of $x_{i}^{(1)}(t) \sim x_{i}^{(4)}(t)$ and trajectories generated by the formation control strategy in the existing result [25], respectively. From these figures, it can be seen that the consensus is also achieved. However, we find that the time-histories of state variables in Case 2 are very oscillated comparing with the result of Case 1. This result indicates that the controller of the existing result [25] cannot flexibly react for the effect of uncertainty.

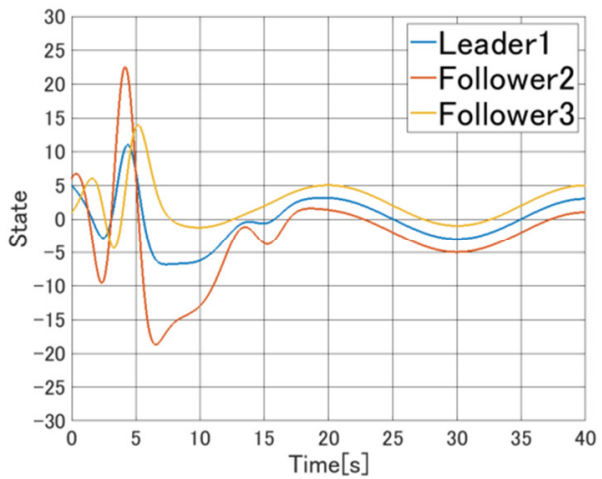

Fig. 7 Time-histories of $x_{i}^{(1)}(t)$ in Case 2

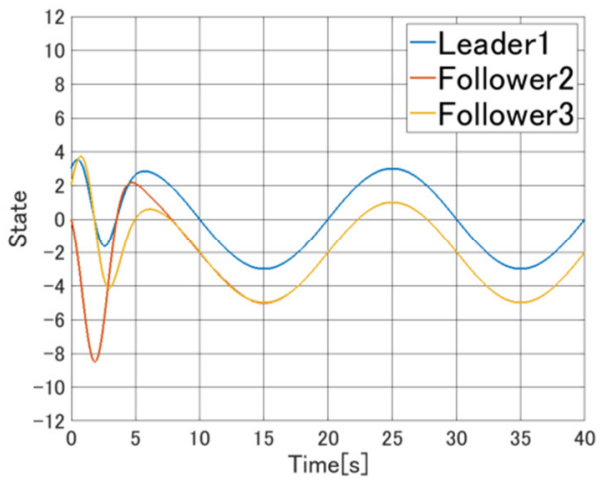

Fig. 9 Time-histories of $x_{i}^{(3)}(t)$ in Case 2

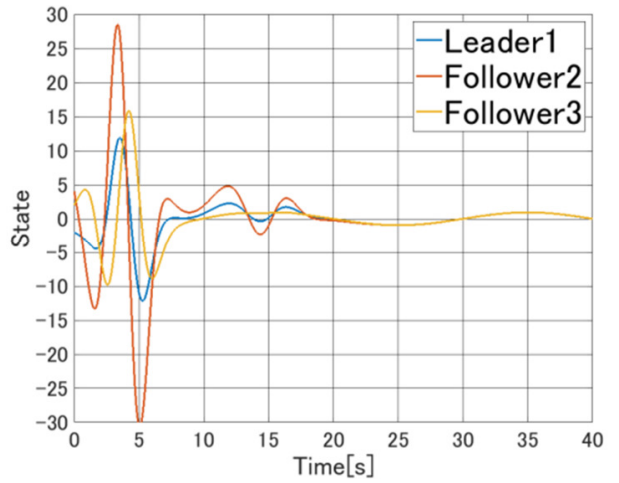

Fig. 8 Time-histories of $x_{i}^{(2)}(t)$ in Case 2

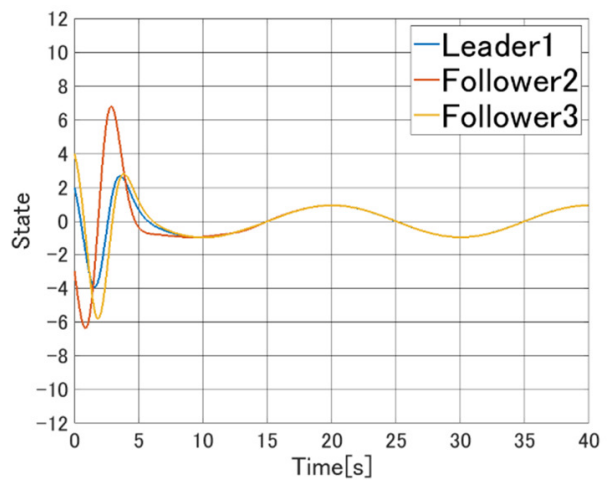

Fig. 10 Time-histories of $x_{i}^{(4)}(t)$ in Case 2
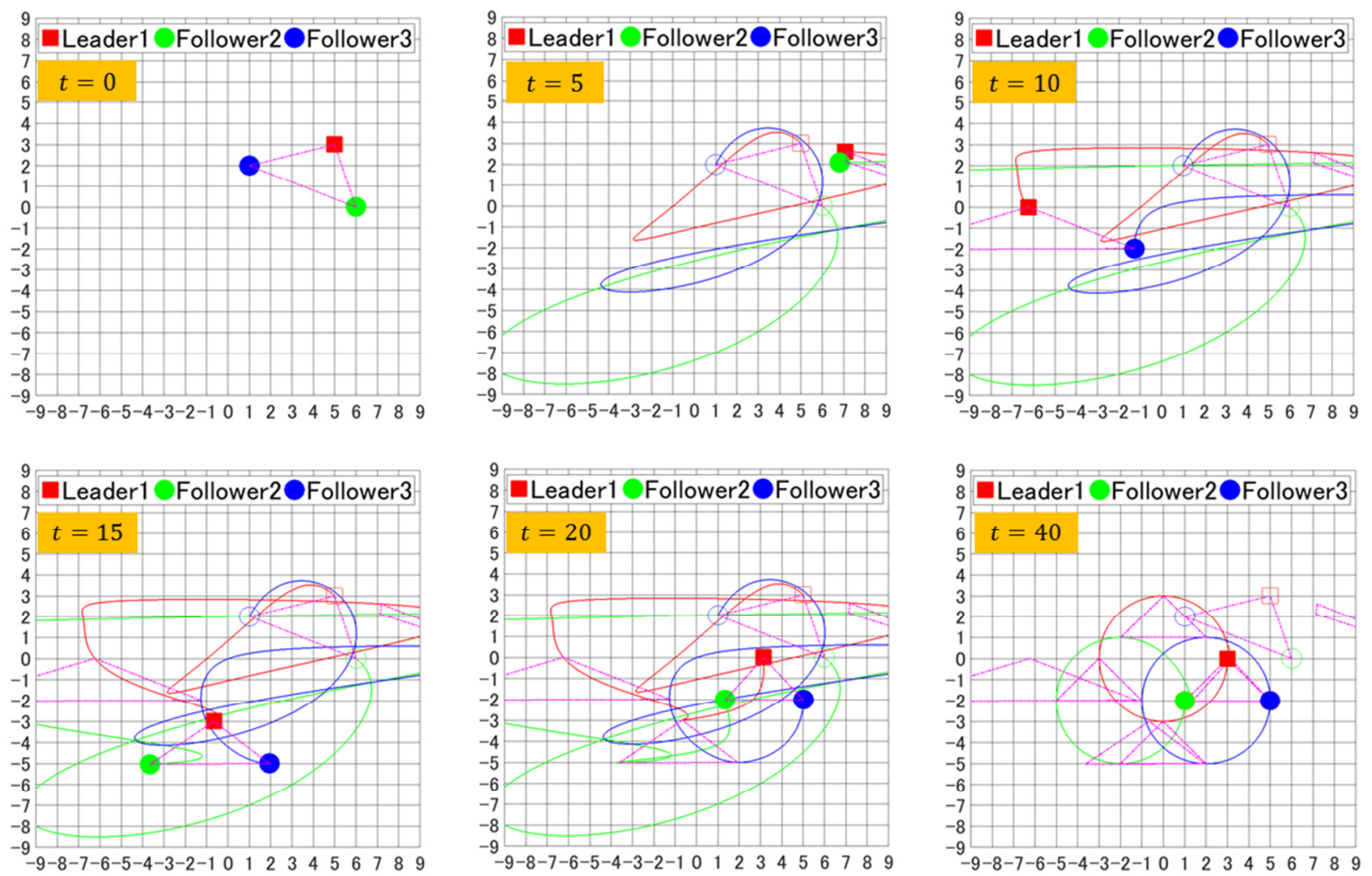

Fig. 11 Trajectories $(x-y$ plane $)$ of MASs in Case 2 
Next, we show the simulation results for the "fixed" gain robust controller, and the time-histories and state trajectories for Case 3 are shown in Figs. 12-16. One can easily see from Figs. 12-16 that the fixed gain robust controller can also achieve consensus. However, it can also be seen that the "fixed" gain robust controller takes a lot of time to perform the formation comparing with the result of Case 1 . This indicates that the proposed adaptive gain robust controller is more flexible and adaptive than the "fixed" gain robust controller.

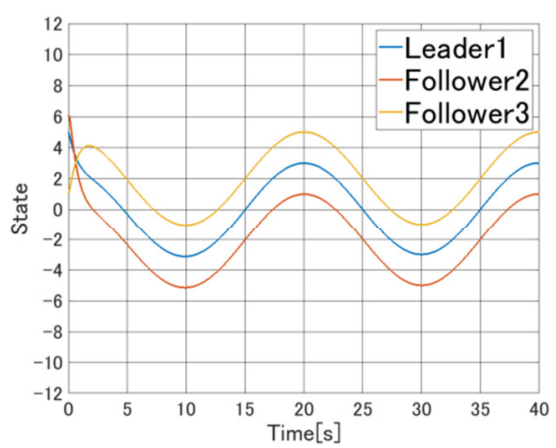

Fig. 12 Time-histories of $x_{i}^{(1)}(t)$ in Case 3

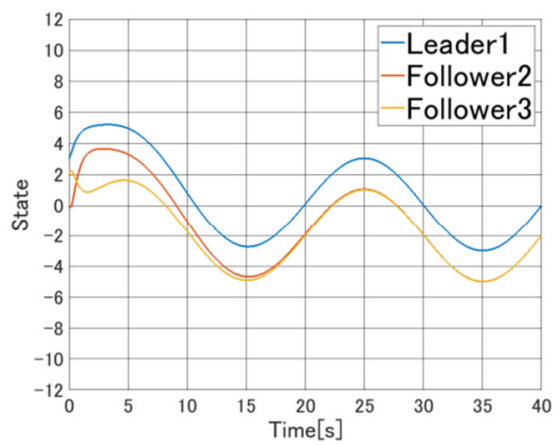

Fig. 14 Time-histories of $x_{i}^{(3)}(t)$ in Case 3

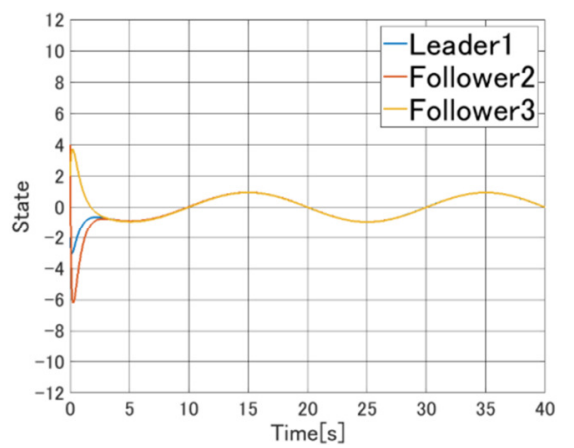

Fig. 13 Time-histories of $x_{i}^{(2)}(t)$ in Case 3

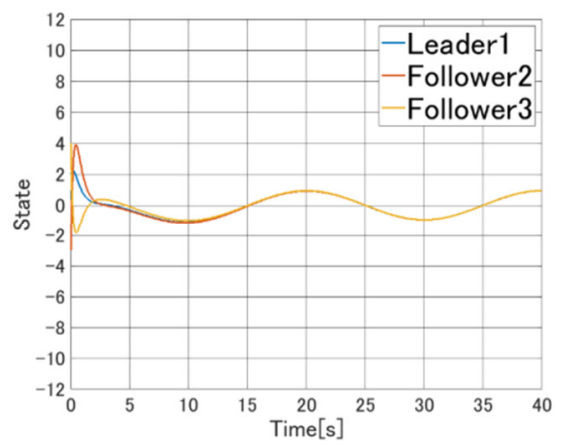

Fig. 15 Time-histories of $x_{i}^{(4)}(t)$ in Case 3
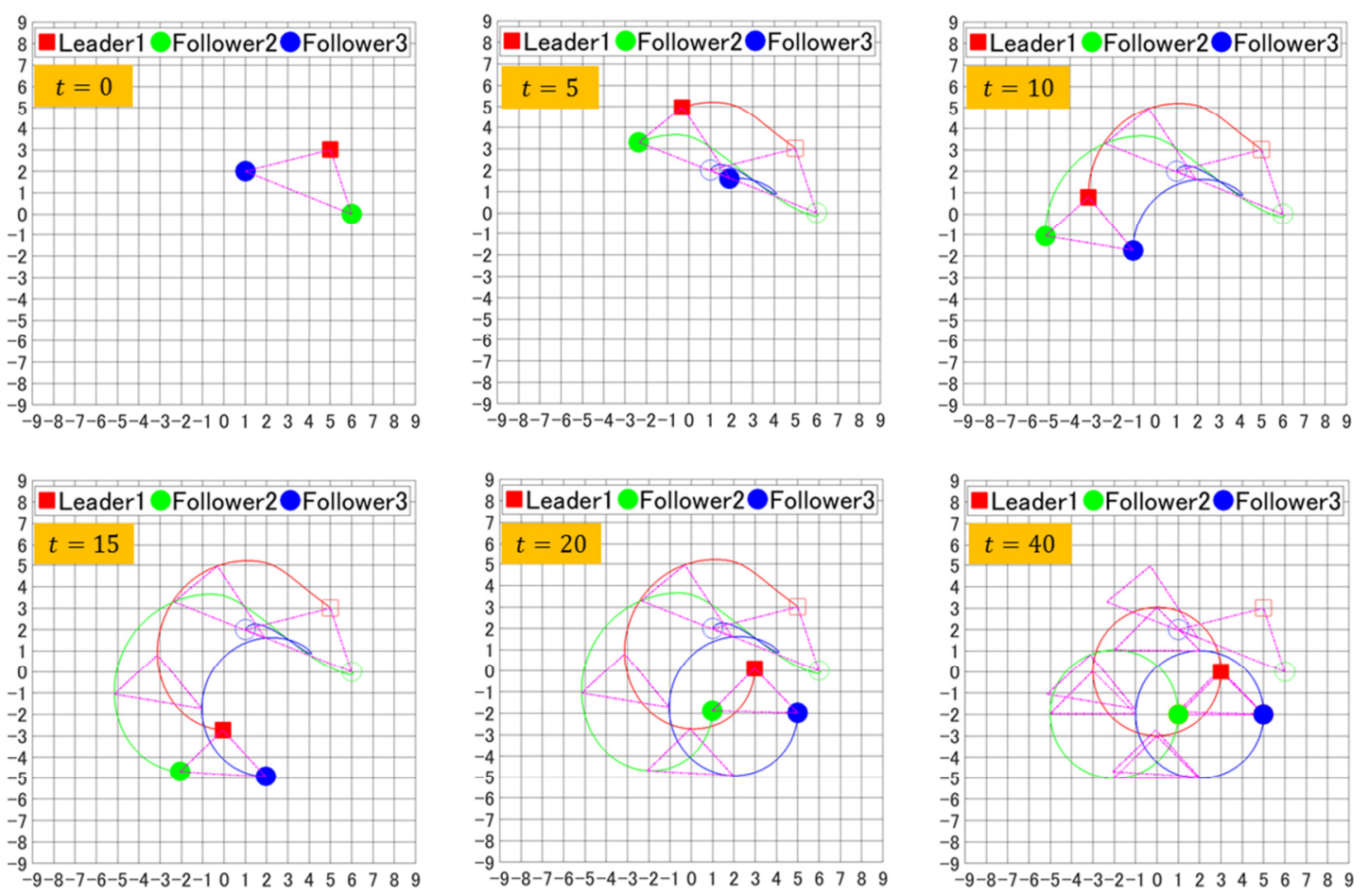

Fig. 16 Trajectories $(x-y$ plane) of MASs in Case 3

To analyze the above results more deeply, the time-histories of the norm of errors between the desired trajectories and the realized responses for each agent in Case 1, Case 2, and Case 3 are presented. In this numerical example, the norm of errors between the desired trajectory and responses of each agent is given by: 


$$
e_{x}(t)=\left\|x(t)-\left(\begin{array}{c}
r(t) \\
r(t)+d_{2} \\
r(t)+d_{3}
\end{array}\right)\right\|
$$

and its time-histories of $e_{x}(t)$ are shown in Fig. 17. In Fig. 17, "Proposed" (resp. "Conventional" and "Robust") means the time-histories of norm of errors for Case 1 (resp. Case 2 and Case 3). One can see from Fig. 17 that the proposed adaptive gain robust controller can smoothly and speedily perform the formation comparing with "Conventional" and "Robust".

From the above discussions, it can be concluded that the consensus has been achieved via the proposed adaptive gain robust controller, and the effectiveness of the proposed formation control strategy has been shown.

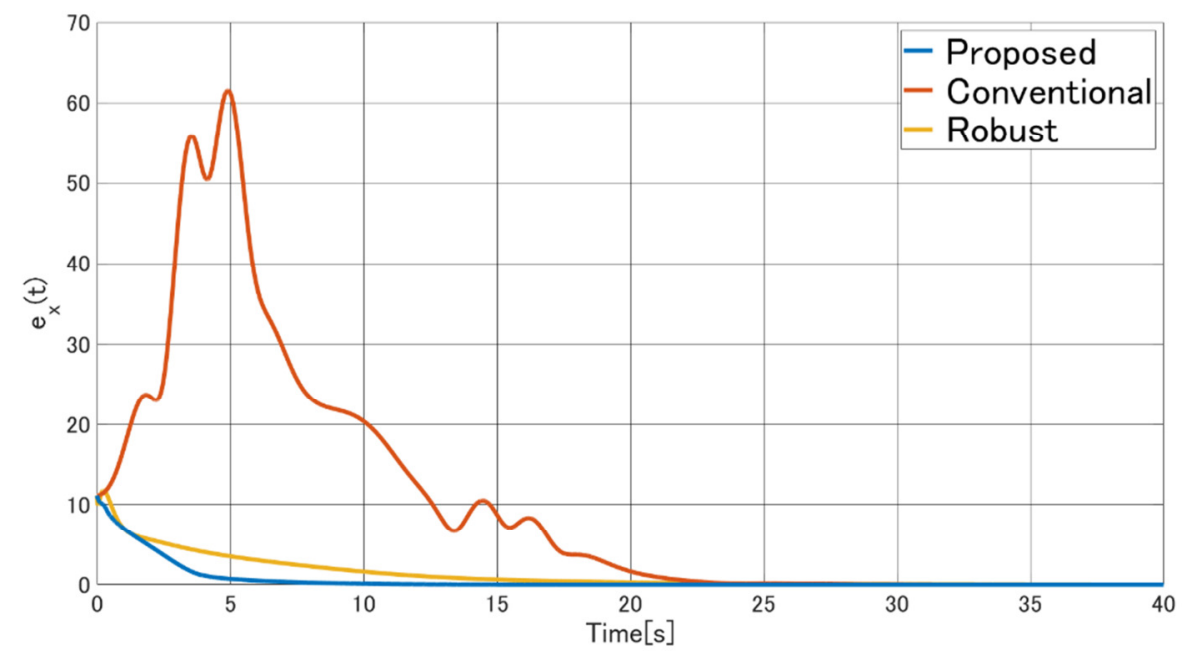

Fig. 17 Time-histories of $e_{x}(t)$

\section{Conclusions}

In this paper, we propose a formation control system via an adaptive gain robust controller which achieves consensus for MASs with uncertainties. In addition, the effectiveness of the proposed robust control system is shown through simple numerical simulations. From the discussions in numerical simulations for uncertain MASs, we find that the proposed adaptive gain robust controller can flexibly and smoothly achieve consensus in spite of uncertainties.

In the proposed robust control strategy, the proposed adaptive gain robust controller consists of fixed feedback gains and variable compensation input tuned by updating rules, and both relative distances between agents and uncertainties are treated explicitly. The sufficient condition for the existence of the proposed formation control system can be reduced to the solvability of LMI, and this fact means that the proposed adaptive gain robust controller can easily be derived by solving LMIs. Furthermore, the proposed robust control strategy only requires the information about the upper bound on relative positions between agents, i.e. there is no need to have the complete information on the target relative position of the other followers. Thus, one can easily see that the result of this paper is a natural extension of the existing result [25].

While the proposed control strategy has some of the advantages mentiond above, the achievable control performance such as transient performance, disturbance attenuation level and so on has not been discussed. Therefore, it will be necessary to tackle design problems for robust formation controllers with achievable performance. In other words, we will extend the proposed robust control strategy to robust consensus controllers which minimize some additional performance indexes such as $L_{2}$ gain performance, quadratic cost functional and so on. Furthermore, extensions of the proposed adaptive gain robust controller to discrete-time systems, time-delay systems, and output feedback control systems are also future research subjects. 


\section{Acknowledgement}

The authors would like to thank Prof. Wen-Hsiang Hsieh, Taiwan Association of Engineering and Technology Innovation and the anonymous reviewers for their valuable and helpful comments that greatly contributed to this paper.

\section{Conflicts of Interest}

The authors declare no conflict of interest.

\section{References}

[1] M. Norton, Modern Control Engineering, New York: Pergamon Press, Inc., 1972.

[2] B. D. Anderson and J. B. Moore, Optimal Control: Linear Quadratic Method, Upper Saddle River: Prentice-Hall, Inc., 1990.

[3] K. Zhou and J. C. Doyle, Essentials of Robust Control, Upper Saddle River: Prentice-Hall, Inc., 1998.

[4] B. Barmish, "Stabilization of Uncertain Systems via Linear Control," IEEE Transactions on Automatic Control, vol. 28, no. 8, pp. 848-850, August 1983.

[5] I. R. Petersen and C. V. Hollot, "A Riccati Equation Approach to the Stabilization of Uncertain Linear Systems," Automatica, vol. 22, no. 4, pp. 397-411, July 1986.

[6] W. E. Schmitendorf, "Designing Stabilizing Controllers for Uncertain Systems Using the Riccati Equation Approach," IEEE Transactions on Automatic Control, vol. 33, no. 4, pp. 376-379, April 1988.

[7] P. P. Khargonekar, I. R. Petersen, and K. Zhou, "Robust Stabilization of Uncertain Linear Systems: Quadratic Stabilizability and $H^{\infty}$ Control Theory," IEEE Transactions on Automatic Control, vol. 35, no. 3, pp. 356-361, March 1990.

[8] I. R. Petersen and D. C. McFarlane, "Optimal Guaranteed Cost Control and Filtering for Uncertain Linear Systems," IEEE Transactions on Automatic Control, vol. 39, no. 9, pp. 1971-1977, September 1994.

[9] M. Maki and K. Hagino, "Robust Control with Adaptation Mechanism for Improving Transient Behaviour," International Journal of Control, vol. 72, no. 13, pp. 1218-1226, 1999.

[10] H. Oya and K. Hagino, "Robust Control with Adaptive Compensation Input for Linear Uncertain Systems," IEICE Transactions on Fundamentals of Electronics, Communications and Computer Sciences, vol. E86-A, no. 6, pp. 1517-1524, June 2003.

[11] H. Oya and K. Hagino, "Adaptive Robust Control Scheme for Linear Systems with Structured Uncertainties," IEICE Transactions on Fundamentals of Electronics, Communications and Computer Sciences, vol. E87-A, no. 8, pp. 2168-2173, August 2004.

[12] D. D. Šiljak, Decentralized Control of Complex Systems, San Diego: Academic Press, Inc., 1991.

[13] L. Lilei, G. Liqun, and Z. Siying, "Decentralized Robust Output Tracking for Large-Scale Systems with Time-Varying Uncertainties," Proceedings of 35th IEEE Conference on Decision and Control, December 1996, pp. 2004-2005.

[14] H. Mukaidani, Y. Takato, Y. Tanaka, and K. Mizukami, "The Guaranteed Cost Control for Uncertain Large-Scale Interconnected Systems,” IFAC Proceedings Volumes, vol. 35, no. 1, pp. 265-270, 2002.

[15] W. J. Mao and J. Chu, "Robust Decentralized Stabilisation of Interval Discrete-Time Singular Large-Scale Systems," IET Control Theory and Applications, vol. 4, no. 2, pp. 244-252, February 2010.

[16] S. Nagai, H. Oya, T. Kubo, and T. Matsuki, "Decentralised Variable Gain Robust Controller Design for a Class of Large-Scale Interconnected Systems with Mismatched Uncertainties,” International Journal of Systems Science, vol. 48, no. 8, pp. 1616-1623, 2017.

[17] R. Olfati-Saber and R. M. Murray, "Consensus Problems in Networks of Agents with Switching Topology and Time-Delays," IEEE Transactions on Automatic Control, vol. 49, no. 9, pp. 1520-1533, September 2004.

[18] G. Xie and L. Wang, "Consensus Control for a Class of Networks of Dynamic Agents: Fixed Topology," Proceedings of the 44th IEEE Conference on Decision and Control, December 2005, pp.96-101.

[19] Y. Zhang and Y. P. Tian, "Consentability and Protocol Design of Multi-Agent Systems with Stochastic Switching Topology,” Automatica, vol. 45, no. 5, pp. 1195-1201, May 2009.

[20] G. Zhai, S. Okuno, J. Imae, and T. Kobayashi, "A Matrix Inequality Based Design Method for Consensus Problems in Multi-Agent Systems," International Journal of Applied Mathematics and Computer Science, vol. 19, no. 4, pp. 639-646, December 2009. 
[21] Y. Y. Qian, L. Liu, and G. Feng, "Distributed Event-Triggered Adaptive Control for Consensus of Linear Multi-Agent Systems with External Disturbances," IEEE Transactions on Cybernetics, vol. 50, no. 5, pp. 2197-2208, May 2020.

[22] W. He, B. Xu, Q. L. Han, and F. Qian, "Adaptive Consensus Control of Linear Multiagent Systems with Dynamic Event-Triggered Strategies,” IEEE Transactions on Cybernetics, vol. 50, no. 7, pp. 2996-3008, July 2020.

[23] X. Xue and F. Wu, "Distributed Consensus Control for General Uncertain Linear Multi-Agent Systems," 37th Chinese Control Conference, July 2018, pp. 7007-7012.

[24] K. Miyakoshi, S. Ito, H. Oya, Y. Hoshi, and S. Nagai, "Synthesis of Formation Control Systems for Multi-Agent Systems under Control Gain Perturbations," Advances in Technology Innovation, vol. 5, no. 2, pp. 112-125, April 2020.

[25] S. Ito, K. Miyakoshi, H. Oya, Y. Hoshi, and S. Nagai, "Consensus via Adaptive Gain Controllers Considering Relative Distances for Multi-Agent Systems," Advances in Technology Innovation, vol. 4, no. 4, pp. 234-246, October 2019.

[26] T. Namerikawa, "Control Theoretical Approach to Multi-Agent Problems," IEICE Technical Report, vol. 108, no. 54, May 2008. (In Japanese)

[27] H. Oya, K. Hagino, and H. Mukaidani, "Robust Non-Fragile Controllers for Uncertain Linear Continuous-Time Systems," 31st Annual Conference of IEEE Industrial Electronics Society, November 2005, pp. 1-6.

[28] S. Boyd, L. El Ghaoui, E. Feron, and V. Balakrishnan, Linear Matrix Inequalities in System and Control Theory, Philadelphia: The Society for Industrial and Applied Mathematics, 1994.

Copyright $\odot$ by the authors. Licensee TAETI, Taiwan. This article is an open access article distributed under the terms and conditions of the Creative Commons Attribution (CC BY-NC) license (https://creativecommons.org/licenses/by-nc/4.0/).

\section{Appendix}

In this Appendix, an LMI-based design method of the "fixed" gain robust controller based on quadratic stabilization [4-5] is shown, i.e. we show a design method of the formation control strategy with fixed gains only. Note that the number of agents is 3. For uncertain MASs of Eq. (5), the control input is defined as

$$
u(t) \triangleq K_{3} \bar{x}(t)+(\mathcal{L} \otimes F) \bar{x}(t)
$$

In Eq. (A.1), the matrices $K$ (resp. $F$ ) is the feedback gain (resp. the consensus gain) for the fixed gain robust controller. From Eqs. (9) and (A.1), one can derive the following uncertain closed-loop system:

$$
\begin{aligned}
\frac{d}{d t} \bar{x}(t) & =\left(I_{3} \otimes \bar{A}\right) \bar{x}(t)+\left(I_{N} \otimes B\right) u(t) \\
& =\left[\bar{A}_{3}+B_{3} K_{3}+B_{3}(\mathcal{L} \otimes F)\right] \bar{x}(t)
\end{aligned}
$$

Therefore, the controller design objective for the fixed gain robust controller is to design the gains $K \in \mathbb{R}^{2 \times 4}$ and $F \in \mathbb{R}^{2 \times 4}$ such that asymptotical stability of the closed-loop system of Eq. (A.2) is ensured.

Now, we introduce the following Lyapunov function candidate:

$$
\mathcal{V}(\bar{x}, t)=\bar{x}^{T}(t)\left(I_{3} \otimes P\right) \bar{x}(t)
$$

Then a condition for asymptotical stability of the closed-loop system of Eq. (A.2) can be written as:

$$
\begin{aligned}
\frac{d}{d t} V(\bar{x}, t)= & \bar{x}^{T}(t)\left[\left\{A_{3}+B_{3} K_{3}+B_{3}(\mathcal{L} \otimes F)\right\}^{T} P_{3}+P_{3}\left\{A_{3}+B_{3} K_{3}+B_{3}(\mathcal{L} \otimes F)\right\}\right] \bar{x}(t) \\
& +\bar{x}^{T}(t)\left\{\left(I_{3} \otimes \Delta(t) E\right)^{T} B_{3}^{T} P_{3}+P_{3} B_{3}\left(I_{3} \otimes \Delta(t) E\right)\right\} \bar{x}(t) \\
& <0
\end{aligned}
$$

By using the well-known that the following relation [27] for matrices $X$ and $Y$ which have appropriate dimensions and a positive scalar $\gamma$, holds: 


$$
X Y+Y^{T} X^{T} \leq \gamma X X^{T}+\frac{1}{\gamma} Y^{T} Y
$$

and by introducing the matrix $E_{3}=\left(I_{3} \otimes P\right)$, we have the following inequality for the second term in Eq. (A.4):

$$
\bar{x}^{T}(t)\left\{\left(I_{3} \otimes \Delta(t) E\right)^{T} B_{3}^{T} P_{3}+P_{3} B_{3}\left(I_{3} \otimes \Delta(t) E\right)\right\} \bar{x}(t) \leq \gamma \bar{x}^{T}(t) P_{3} B_{3} B_{3}^{T} P_{3} \bar{x}(t)+\frac{1}{\gamma} \bar{x}^{T}(t) E_{3}{ }^{T} E_{3} \bar{x}(t)
$$

Therefore, one can see from Eqs. (A.4) and (A.6) that the sufficient condition for asymptotical stability of the closed-loop system of Eq. (A.2) can be derived as:

$$
\begin{aligned}
\frac{d}{d t} V(\bar{x}, t) & \leq \bar{x}^{T}(t)\left[\left\{A_{3}+B_{3} K_{3}+B_{3}(\mathcal{L} \otimes F)\right\}^{T} P_{3}+P_{3}\left\{A_{3}+B_{3} K_{3}+B_{3}(\mathcal{L} \otimes F)\right\}\right] \bar{x}(t) \\
& +\gamma \bar{x}^{T}(t) P_{3} B_{3} B_{3}^{T} P_{3} \bar{x}(t)+\frac{1}{\gamma} \bar{x}^{T}(t) E_{3}{ }^{T} E_{3} \bar{x}(t) \\
& <0
\end{aligned}
$$

Namely, if the inequality is satisfied as:

$$
\left\{A_{3}+B_{3} K_{3}+B_{3}(\mathcal{L} \otimes F)\right\}^{T} P_{3}+P_{3}\left\{A_{3}+B_{3} K_{3}+B_{3}(\mathcal{L} \otimes F)\right\}+\gamma P_{3} B_{3} B_{3}^{T} P_{3}+\frac{1}{\gamma} E_{3}^{T} E_{3}<0
$$

then the inequality condition of Eq. (A.3) is also ensured, and the asymptotic stability for the closed-system of Eq. (A.2) is guaranteed.

Here, in order to determine the gain matrices $K \in \mathbb{R}^{2 \times 4}$ and $F \in \mathbb{R}^{2 \times 4}$, a symmetric positive definite matrix $S=P^{-1}$ and complementary variables $W_{K} \triangleq K S$ and $W_{F} \triangleq F S$ are introduced. Moreover, pre- and post-multiplying Eq. (A.8) by $S_{3}=$ $\left(I_{3} \otimes S\right)$, the following inequality for the asymptotic stability for the closed-system of Eq. (A.2) are obtained:

$$
\begin{aligned}
& \left(\mathcal{L} \otimes W_{F}\right)^{T} B_{3}^{T}+\left(I_{3} \otimes W_{K}\right)^{T} B_{3}^{T}+S_{3} A_{3}^{T}+A_{3} S_{3}+B_{3}\left(I_{3} \otimes W_{K}\right) \\
& +B_{3}\left(\mathcal{L} \otimes W_{F}\right)+\gamma B_{3} B_{3}^{T}+\frac{1}{\gamma} S_{3} E_{3}^{T} E_{3} S_{3}<0
\end{aligned}
$$

Furthermore, by applying Schur complement formula (see [28] for details) to the inequality of Eq. (A.9), we find that the inequality condition of Eq. (A.9) is equivalent to:

$$
\left(\begin{array}{c:c}
\Lambda\left(S, W_{K}, W_{F}, \gamma\right) & S_{3} E_{3}{ }^{T} \\
\hdashline E_{3} S_{3} & -\gamma I_{6}
\end{array}\right)<0
$$

where the matrix $\Lambda\left(S, W_{K}, W_{F}, \gamma\right)$ is given as:

$$
\Lambda\left(S, W_{K}, W_{F}, \gamma\right)=\left(\begin{array}{ccc}
\Lambda_{11}\left(S, W_{K}, \gamma\right) & \Lambda_{12}\left(W_{F}\right) & \Lambda_{13}\left(W_{F}\right) \\
\star & \Lambda_{22}\left(S, W_{K}, W_{F}, \gamma\right) & \Lambda_{23}\left(W_{F}\right) \\
\star & \star & \Lambda_{33}\left(S, W_{K}, W_{F}, \gamma\right)
\end{array}\right)
$$

In (A.11), $\Lambda_{i j}\left(S, W_{K}, W_{F}, \gamma\right)$ are matrices given by:

$$
\begin{aligned}
& \Lambda_{11}\left(S, W_{K}, \gamma\right)=S A^{T}+W_{K}^{T} B^{T}+A S+B W_{K}+\gamma B B^{T} \\
& \Lambda_{22}\left(S, W_{K}, W_{F}, \gamma\right)=\Lambda_{33}\left(S, W_{K}, W_{F}, \gamma\right)=S A^{T}+W_{K}^{T} B^{T}+2 W_{F}^{T} B^{T}+A S+B W_{K}+2 B W_{F}+\gamma B B^{T} \\
& \Lambda_{12}\left(W_{F}\right)=\Lambda_{13}\left(W_{F}\right)=-W_{F}^{T} B^{T} \\
& \Lambda_{23}\left(W_{F}\right)=-\left(W_{F}^{T} B^{T}+B W_{F}\right)
\end{aligned}
$$


Namely, the inequality of Eq. (A.10) is a linear matrix inequality (LMI) for the matrices $S, W_{K}, W_{F}$ and a positive scalar $\gamma$. If the solution of Eq. (A.10) exists, then the closed-loop system of Eq. (A.2) is asymptotically stable, and formation/consensus can be achieved. Additionally, by using, $S \in \mathbb{R}^{4 \times 4}, W_{K} \in \mathbb{R}^{2 \times 4}$, and $W_{F} \in \mathbb{R}^{2 \times 4}$, which satisfy the LMI of Eq. (A.10), the gain matrices $K \in \mathbb{R}^{2 \times 4}$ and $F \in \mathbb{R}^{2 \times 4}$ for the fixed gain robust controller can be derived as:

$$
K=W_{K} S^{-1}, F=W_{F} S^{-1}
$$

Consequently, we have the following theorem for the "fixed" gain robust controller based on quadratic stabilization:

Theorem A.1: Consider the uncertain closed-loop system of Eq. (A.2). If there exist solutions $S \in \mathbb{R}^{4 \times 4}, W_{K} \in \mathbb{R}^{2 \times 4}$, $W_{F} \in \mathbb{R}^{2 \times 4}$, and a positive scalar $\gamma$ which satisfy the linear matrix inequality (LMI):

$$
\begin{aligned}
& \left(\begin{array}{ccc:c}
\Lambda_{11}\left(S, W_{K}, \gamma\right) & \Lambda_{12}\left(W_{F}\right) & \Lambda_{13}\left(W_{F}\right) & \\
\star & \Lambda_{22}\left(S, W_{K}, W_{F}, \gamma\right) & \Lambda_{23}\left(W_{F}\right) & S_{3} E_{3}^{T} \\
\star & \star & \Lambda_{33}\left(S, W_{K}, W_{F}, \gamma\right) & \\
\hdashline E_{3} S_{3} & & -\gamma I_{6}
\end{array}\right)<0 \\
& \Lambda_{11}\left(S, W_{K}, \gamma\right)=S A^{T}+W_{K}^{T} B^{T}+A S+B W_{K}+\gamma B B^{T} \\
& \Lambda_{22}\left(S, W_{K}, W_{F}, \gamma\right)=\Lambda_{33}\left(S, W_{K}, W_{F}, \gamma\right)=S A^{T}+W_{K}^{T} B^{T}+2 W_{F}^{T} B^{T}+A S+B W_{K}+2 B W_{F}+\gamma B B^{T} \\
& \Lambda_{12}\left(W_{F}\right)=\Lambda_{13}\left(W_{F}\right)=-W_{F}^{T} B^{T}, \Lambda_{23}\left(W_{F}\right)=-\left(W_{F}^{T} B^{T}+B W_{F}\right)
\end{aligned}
$$

then asymptotic stability for the closed-loop system of Eq. (A.2) is ensured, and the consensus for MASs of Eq. (9) can be achieved. Moreover, the stabilizing feedback gain $K \in \mathbb{R}^{2 \times 4}$ and the consensus one $F \in \mathbb{R}^{2 \times 4}$ can be calculated as:

$$
K=W_{K} S^{-1}, \quad F=W_{F} S^{-1}
$$

Remark A.1: In the "fixed" gain robust control based on quadratic stabilization [4-5], the control law is given as Eq. (A.1), and it can be rewritten as:

$$
u(t)=\left(\begin{array}{l}
u_{1}(t) \\
u_{2}(t) \\
u_{3}(t)
\end{array}\right)=\left(\begin{array}{c}
K\left(x_{l}(t)-d_{l}\right) \\
-F\left(x_{l}(t)-d_{l}\right)+(K+2 F)\left(x_{2}(t)-d_{2}\right)-F\left(x_{3}(t)-d_{3}\right) \\
-F\left(x_{l}(t)-d_{l}\right)-F\left(x_{2}(t)-d_{2}\right)+(K+2 F)\left(x_{3}(t)-d_{3}\right)
\end{array}\right)
$$

One can easily see from Eq. (A.16) that the "fixed" gain robust controller needs the exact information for relative positions for the other agents. Thus, if there exist uncertainties for the information for relative positions, then the "fixed" gain robust controller cannot achieve the desired formation. On the other hand, the proposed adaptive gain robust controller utilizes the information about the upper bound on relative positions only. From this point, the proposed control strategy is more flexible than the "fixed" gain robust controller. 